far more regularly than this (daily, if necessary). While this might seem excessive, it appears that it is crucial to maintaining such patients in the community, and is ultimately cost-effective. Indeed, Preston \& Fazio (2000) showed that for our ICM service, with a capped case-load per case worker of around 10 patients, and a mean number of annual community contacts of 164 (s.d.=20) v. 56 (s.d.=100) for nonintensive patients, in-patient bed-days fell dramatically (from a mean of 118 days (s.d.=113) per year before ICM, to 57 days (s.d.=91) in the second year of the ICM intervention). The control group showed no such reduction in bed-days, and the overall cost saving (factoring in the increased out-patient costs for the ICM group) at the end of the 2 years was AU\$801 475 for 65 patients $(P<0.001)$.

Thus, it is important that the precise nature of the intervention is examined before dismissing ICM as a cost-effective model of service delivery.

Preston, N. J. \& Fazio, S. (2000) Establishing the efficacy and cost effectiveness of community intensive case management of long-term mentally ill: a matched control group study. Australian and New Zealand Journal of Psychiatry, 34, I14-121.

UK700 Group (2000) Cost-effectiveness of intensive v. standard case management for severe psychotic illness. UK700 case management trial. British Journal of

Psychiatry, 176, 537-543.

D. J. Castle University of Western Australia and Fremantle Hospital \& Health Service, Alma Street, Fremantle 6160,Western Australia

We read with interest the paper regarding the cost-effectiveness of intensive $v$. standard case management for severe psychotic illness (UK700 Group, 2000).

We feel very strongly that more comment should have been made on the topic of training (or lack of training) of the case managers involved. The findings of this large, well-designed trial are very similar to those of Muijen et al (1994), who found no difference in outcome between the use of community psychiatric nurses (CPNs) configured into case management teams $v$. CPNs working in a generic way. The main implication of this study was that merely reconfiguring services into different working arrangements provides no additional benefits to patients. However, it could be argued that benefits may accrue if training in research-based interventions is provided. Indeed, such training for CPNs and other health care professionals has been developed in the Thorn and similar programmes, which focus specifically on providing skills in assertive community treatment, family interventions, psychological interventions etc. One could argue that these groups of skills, which comprise what is loosely known as psychosocial interventions, are essential to effective case management.

In the UK700 study, we note that the case managers received a 2-day induction course in case management and an unspecified amount of instruction in outreach practice given by a team leader in the assertive community treatment service from Boulder, Colorado. It seems to us that such training input is insufficient to provide the skills necessary to deliver truly effective psychosocial care. (The Thorn programme comprises 36 days of direct training plus the equivalent of 50 days of further study and project work.) We are therefore not surprised that the case managers with smaller case-load sizes could not improve on the outcomes attained by those working with the more average size case-loads.

Surely studies of training per se are now needed, with random allocation of case managers to training in research-based interventions or to standard practice, and measurement of outcomes for both the trainees (in terms of skills acquisition and knowledge gain) and their patients (in terms of clinical outcomes).

We are at present spending enormous amounts of money on training throughout the National Health Service and yet the vast majority of this training remains completely unevaluated. Although randomised controlled trials of training interventions will be costly, the price of not knowing whether training makes a difference is much greater.

Muijen, M., Cooney, M., Strathdee, G., et al (1994) Community psychiatric nurse teams: intensive support versus generic care. British Journal of Psychiatry, 165 , $211-217$.

UK700 Group (2000) Cost-effectiveness of intensive $v$ standard case management for severe psychotic illness. UK700 case management trial. British journal of Psychiatry, 176, 537-543.

K. Gournay, G. Thornicroft Department of Health Services Research, Institute of Psychiatry, De Crespigny Park, Denmark Hill, London SE5 8AF

\section{Lithium and mortality}

In their study of mortality in patients with affective disorder commenced on lithium, Brodersen et al (2000) paint an unfairly negative picture of the efficacy of lithium.
They compared mortality in patients with affective disorders who were started on lithium, irrespective of their compliance with treatment, with that of the general population. This gives a false impression that lithium could increase mortality. To assess the efficacy of lithium, they should ideally have compared those who were compliant with the treatment with those who were not and also with the general population, as Kallner et al (2000) did. The latter study clearly demonstrates that even though affective disorder patients have an increased mortality compared with the general population, lithium has a definite antisuicidal effect. Moreover, in unipolar depression, suicide rates increased only after patients discontinued lithium. These two studies also show how the methodology can affect the findings.

Brodersen, A., Licht, R. W., Vestergaard, P., et al (2000) Sixteen-year mortality in patients with affective disorder commenced on lithium. British Journal of Psychiatry, 176, 429-433.

Kallner, G., Lindelius, R., Petterson, U., et al (2000) Mortality in 497 patients with affective disorders attending a lithium clinic or after having left it. Pharmacopsychiatry, 33, 8-13.

K. S. Gracious Medway Hospital,Windmill Road, Gillingham, Kent ME7 5NY

F. Falodun West Suffolk Hospital, Hardwick Lane, Bury St Edmunds IP33 2QZ

Authors' reply: Gracious \& Falodun find that our study of mortality in affective disorder patients commenced on lithium (Brodersen et al, 2000) paints an unfairly negative picture of lithium's efficacy. They object to our intention-to-treat analysis of all patients commenced on lithium irrespective of compliance, which showed a significantly elevated standardised mortality ratio (SMR) of 2.5. They suggest that we should have compared compliant with non-compliant patients and with the general population, as did Kallner et al (2000).

Kallner et al actually reported - even in patients compliant with lithium - that mortality in general $(\mathrm{SMR}=1.6)$ and suicide in particular $(\mathrm{SMR}=14.0)$ were significantly elevated. They also found that mortality was even higher in non-compliant patients, a result which may very well be valid. However, comparison of compliant with non-compliant patients introduces a considerable selection bias, since patients are not randomly allocated to the two groups. Rather, patients with comorbidity, such as 